\title{
Measurements of the production of a vector boson in association with jets in the ATLAS and CMS detectors
}

\author{
Vieri Candelise ${ }^{1, a}$ \\ ${ }^{1}$ University of Trieste and INFN Trieste, Via Valerio 2, 34128, Trieste, Italy
}

\begin{abstract}
The production of a vector boson $(\mathrm{V}=\mathrm{W}, \mathrm{Z})$ in association with jets is can be used for a precise test of perturbative QCD and is a dominant background process in many searches for physics beyond the Standard Model. The differential cross section measurements from CMS and ATLAS collaborations would be presented, and several theoretical predictions at leading order (LO) and next-to leading order (NLO) are compared to data, after the deconvolution of detector effects, with proton-proton collisions at a center of mass energy of 7 , 8 and $13 \mathrm{TeV}$ at $\mathrm{LHC}$.
\end{abstract}

\section{Introduction}

The associated production of vector bosons and jets $(\mathrm{V}+\mathrm{jets})$ in proton-proton collisions at LHC is of prime interest in the physics program of the ATLAS and CMS experiments [1,2]. This type of processes is important for several physics aspects. First of all in perturbative QCD (pQCD) studies, where direct measurement of the quark content of the proton and parton density functions (PDF) can be made by measuring the production cross sections. Studying the differential cross sections is also essential to test the latest $\mathrm{pQCD}$ calculations at the highest precision available in the perturbative expansion, making these measurements an unique tool for the test the Monte Carlo event generators. In Standard Model physics, the $\mathrm{W}, \mathrm{Z}$ plus jets production is an essential measurement in order to precisely estimate the background component of the Higgs boson and top pairs production. Furthermore, many Beyond the Standard Model searches (such as supersymmetry, new Higgs bosons, 4th generation of heavy quarks) have a similar topology with respect to the $\mathrm{V}+$ jets production, and thus the control on their backgrounds might be highly improved by means of these measurements.

\section{Associated production of a $\mathbf{W}$ boson and jets}

The associated production of a $\mathrm{W}$ boson and one or more jets has been measured in proton-proton collisions at the center of mass energy of $7 \mathrm{TeV}$ both in ATLAS and CMS. The differential spectra are deconvolved for detector effects and compared to the theoretical predictions at LO and NLO precision.

\subsection{W+jets at $7 \mathrm{TeV}$ in ATLAS}

The $\mathrm{W}+$ jets measurement in ATLAS [3] has been performed using an integrated luminosity of $4.6 \mathrm{fb}^{-1}$. Electrons and muons from the $\mathrm{W}$ boson decay must pass isolation criteria and are required to have a transverse momentum $\left(\mathrm{p}_{\mathrm{T}}\right)$ of at least $25 \mathrm{GeV}$ and pseudorapidity $(\eta)$ less than 2.4 . The reconstructed missing transverse energy is used to account for the presence of the neutrino, and the associated jets are clustered using the anti- $\mathrm{K}_{\mathrm{T}}$ algorithm [4] with $\mathrm{R}=0.4$. Jets must also have $\mathrm{p}_{\mathrm{T}}>25 \mathrm{GeV}$ and rapidity (y) within the range of 4.4. The background coming from the production of top quark pairs is suppressed by requiring a $\mathrm{b}$ quark tag veto, and the multi-jet QCD background is estimated in data. Measured cross sections are unfolded to particle level using a Bayesian algorithm based on the Monte Carlo information. The differential cross sections are presented as a function of the leading jet

a Corresponding author: vieri@CERN.ch 
momentum (Fig.1). Data are compared to the theoretical prediction of BlackHat+Sherpa (NLO) [5], ALPGEN (LO) [6], and Sherpa v.2 [7]. It can be seen that the LO calculations describe data in the hard part of the spectrum within the uncertainties, while a $10 \%$ overestimation is seen in the $200-400$ $\mathrm{GeV}$ range. NLO calculations show good agreement in the comparison with unfolded data.
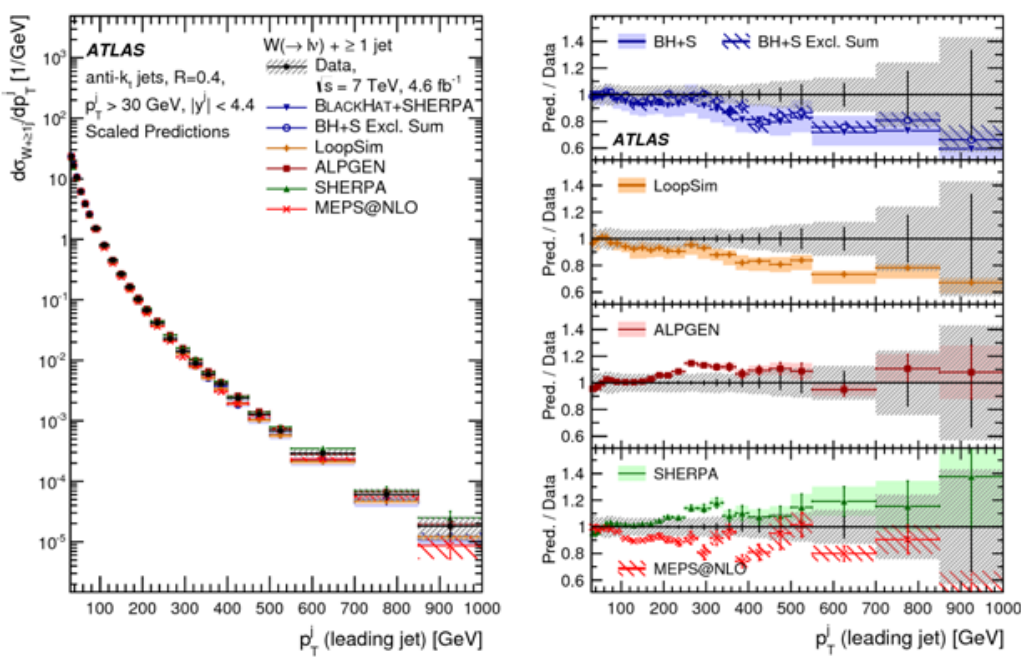

Figure 1. Differential $\mathrm{W}+$ jets cross sections as a function of the leading jet transverse momentum compared to the theoretical predictions of BlackHat+Sherpa at NLO, Sherpa (LO) and MadGraph+Pythia6 (LO) measured in ATLAS.

\subsection{W+jets at $7 \mathrm{TeV}$ in CMS}

The $\mathrm{W}+$ jets cross section in CMS [8] has been measured using an integrated luminosity of $5 \mathrm{fb}^{-1}$. Electrons and muons from the $\mathrm{W}$ boson decay must be isolated and are required to have $\mathrm{p}_{\mathrm{T}}>25 \mathrm{GeV}$ and $|\eta|<2.1$. Associated jets are clustered using the anti-K $\mathrm{K}_{\mathrm{T}}$ algorithm with $\mathrm{R}=0.5$. Jets must also have $\mathrm{p}_{\mathrm{T}}>25 \mathrm{GeV}$ and have $|\eta|<2.4$. A cut on the missing transverse mass $\left(\mathrm{M}_{\mathrm{T}}\right)$ of the neutrino and the muon is applied, requiring $\mathrm{M}_{\mathrm{T}}>50 \mathrm{GeV}$. The background coming from the production of top quark pairs is taken into account requiring a b quark tag veto, and the multi-jet QCD background is estimated again with a data-driven technique. Measured cross sections are unfolded to particle level using the SVD algorithm based on the Monte Carlo simulation. The differential cross sections are presented as a function of the leading jet momentum and as a function of the scalar sum of all the jets in the event, namely $\mathrm{H}_{\mathrm{T}}$ (Fig.2). Data are compared to the theoretical expectations of MadGraph5 [9]+Pythia6 [10] (LO), BlackHat+Sherpa (NLO) and Sherpa (LO). It can be seen that the NLO predictions of BlackHat +Sherpa are not describing properly the spectra of the $\mathrm{H}_{\mathrm{T}}$ distribution. It is shown that the LO MadGraph+Pythia6 prediction is over-estimating data in the full range of $\mathrm{p}_{\mathrm{T}}$ of the leading jet and $\mathrm{H}_{\mathrm{T}}$.

\section{Associated production of a $Z$ boson and jets}

The associated production of a $\mathrm{Z}$ boson and at least one jet measurement in proton-proton collisions at the center of mass energy of $8 \mathrm{TeV}$ in CMS is presented. The differential cross sections are unfolded for detector effects and compared to the theoretical predictions at the LO and NLO precision. 

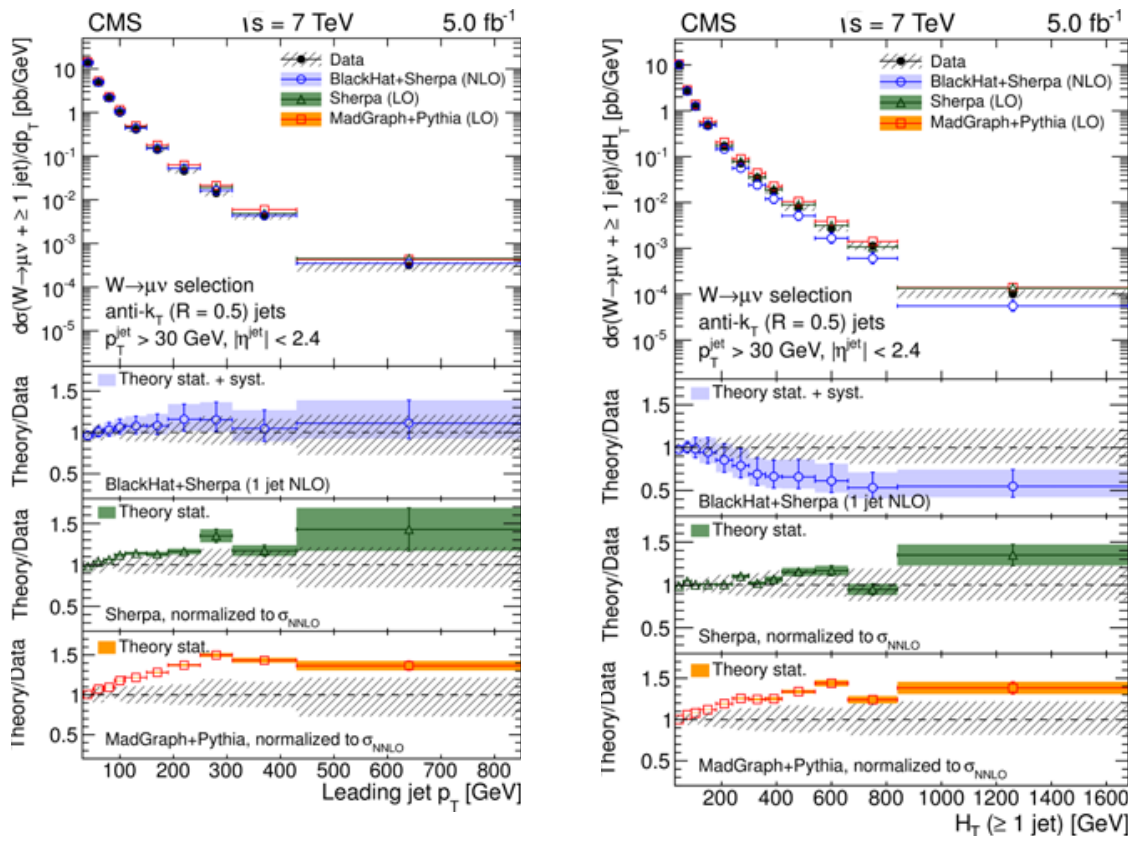

Figure 2. Differential $\mathrm{W}+$ jets cross sections as a function of the leading jet transverse momentum (left) and $\mathrm{H}_{\mathrm{T}}$ (right) compared to the theoretical predictions of BlackHat+Sherpa at NLO, Sherpa (LO) and MadGraph+Pythia6 (LO) measured in CMS.

\subsection{Z+jets differential and double differential cross sections at $8 \mathrm{TeV}$ in CMS}

The $\mathrm{Z}+$ jets differential cross section measurement in CMS [11] has been performed using an integrated luminosity of $5 \mathrm{fb}^{-1}$. Electron and muon pairs from the $\mathrm{Z}$ boson decay must be isolated and must have $\mathrm{p}_{\mathrm{T}}>20 \mathrm{GeV}$ and $|\eta|<2.4$. The dilepton pairs are selected if their invariant mass lies within the mass range of $[71 ; 111] \mathrm{GeV}$. Jets are clustered using the anti-K $\mathrm{K}_{\mathrm{T}}$ algorithm with $\mathrm{R}=0.5$ and must pass a $\mathrm{p}_{\mathrm{T}}>25 \mathrm{GeV}$ threshold, and have $|\eta|<2.4$. Measured cross sections are unfolded to particle level using the Bayesian unfolding algorithm exploiting the simulation information. The differential cross sections are presented as a function of the jet multiplicity (Fig.3). Data are compared to the theoretical expectations of MadGraph5+Pythia6 (LO) and Sherpa v.2 (NLO up to the second jet). Good agreement is seen in the unfolded cross sections. The double differential cross sections has also been measured as a function of the leading jet momentum and several ranges of rapidity, up to $|y|<4.7[6]$.

\subsection{Z+jets differential cross sections at $13 \mathrm{TeV}$ in ATLAS}

The $\mathrm{Z}+$ jets differential cross sections, measured at a center of mass energy of $13 \mathrm{TeV}$, have been studied with the ATLAS detector [12] with an integrated luminosity of $85 \mathrm{pb}^{-1}$. Dielectron (dimuon) pairs coming from the $\mathrm{Z}$ boson decay must be isolated and must have $\mathrm{p}_{\mathrm{T}}>25 \mathrm{GeV}$ and $|\eta|<2.47(2.4)$. The dilepton invariant mass is taken within the mass range of $[66 ; 116] \mathrm{GeV}$. Jets are clustered using anti- $\mathrm{K}_{\mathrm{T}}$ algorithm with $\mathrm{R}=0.4$ and must also have a transverse momentum of at least $30 \mathrm{GeV}$ and $|\mathrm{y}|$ $<2.5$. Measured cross sections are corrected to particle level using a correction factor calculated with the Sherpa event generator. The differential cross sections are presented as a function of the jet multiplicity (Fig.4). Data are compared to the theoretical predictions of MadGraph_aMC@NLO +Pythia8 [13] (up to four partons at LO) and Sherpa v.2 (NLO up to the second jet). A good agreement is seen in the measured cross sections. 


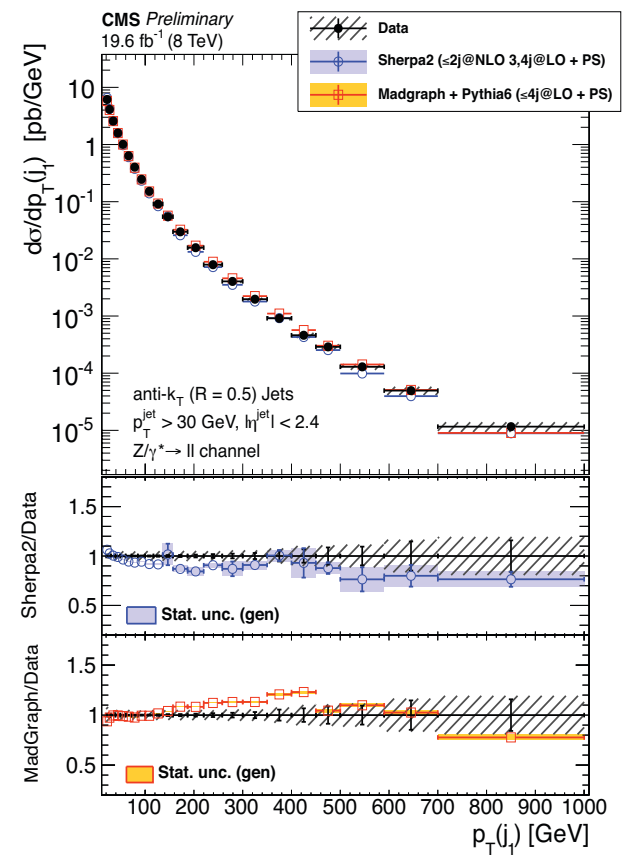

Figure 3. Differential $\mathrm{Z}+\mathrm{jets}$ cross sections as a function of the leading jet transverse momentum compared to the theoretical predictions of Sherpa v2 (NLO up to the second jet) and MadGraph+Pythia6 (LO) measured in CMS.

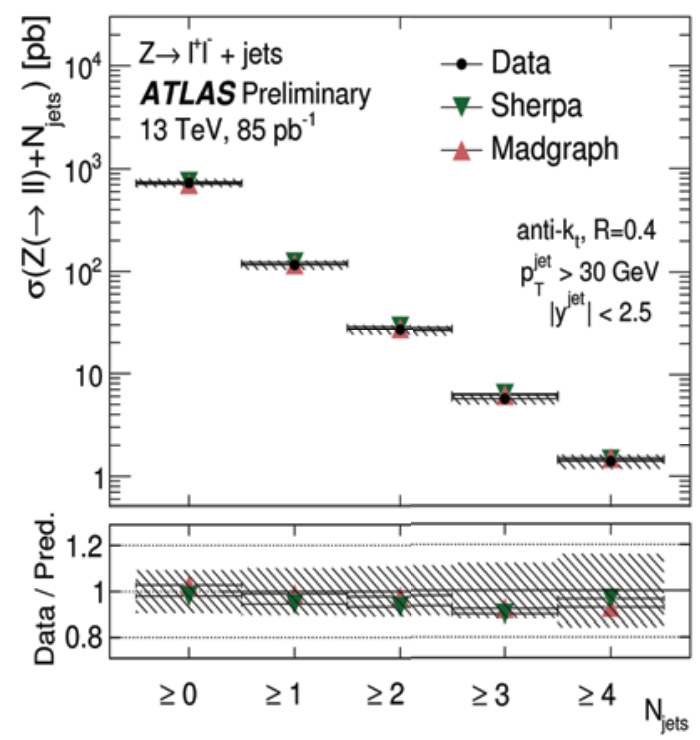

Figure 4. The $13 \mathrm{TeV}$ differential $\mathrm{Z}+$ jets cross sections as a function of the jet multiplicity compared to the theoretical predictions of Sherpa v2 (NLO up to the second jet) and MadGraph_aMC@NLO+Pythia8 (LO) measured with the ATLAS detector. 


\section{Differential W+jets and Z+jets cross-section ratio in ATLAS}

The differential cross section ratio between $\mathrm{W}+$ jets and $\mathrm{Z}+$ jets is an important tool to test the pQCD predictions, exploiting the advantage of many important systematics cancelation in the ratio, thus increasing the precision in the final results. Furthermore, the precise modeling of the jet recoil against the vector boson is an important measurement for many searches for physics beyond the Standard Model. Events are collected using $4.6 \mathrm{fb}^{-1}$ of the $7 \mathrm{TeV}$ data taking of the ATLAS detector [14]. Isolated leptons are selected from the boson decay if they have $\mathrm{p}_{\mathrm{T}}>25 \mathrm{GeV}$ and $|\eta|<2.47$. Associated jets are clustered using the anti- $\mathrm{K}_{\mathrm{T}}$ algorithm with $\mathrm{R}=0.4$. Jets must have $\mathrm{p}_{\mathrm{T}}>25 \mathrm{GeV}$ and $|\mathrm{y}|<4.4$. Additional requirements on the missing transverse energy and mass are imposed for the $\mathrm{W}$ boson reconstruction, and the dilepton pairs are selected in the range of the $\mathrm{Z}$ boson mass according to the $\mathrm{Z}$ +jets selection. Data are unfolded to particle level by means of the Bayesian unfolding procedure. Unfolded data are then compared to the LO predictions of ALPGEN, Sherpa and the NLO prediction of BlackHat+Sherpa. Differential cross section ratios are presented as a function of the leading jet transverse momentum (Fig. 5), showing general good agreement all over the spectrum, but with some tensions that can be seen comparing data to the Sherpa prediction around $300 \mathrm{GeV}$, where the generator overestimates data.

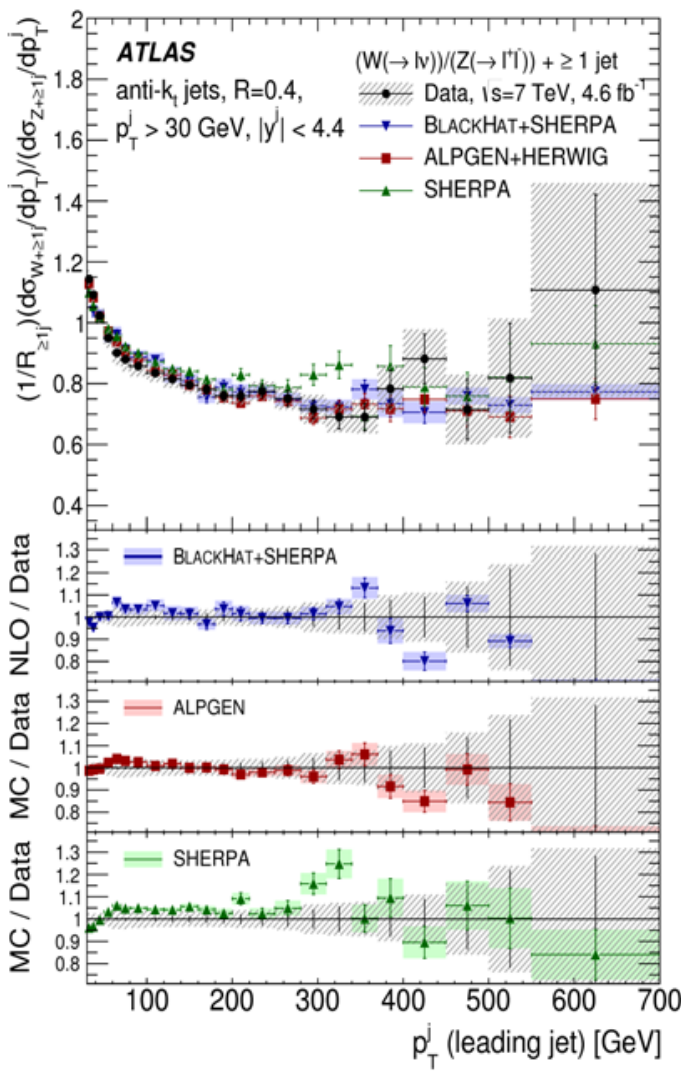

Figure 5. Differential $\mathrm{W}+$ jets/Z+jets cross sections ratio as a function of the leading jet transverse momentum compared to the theoretical predictions of Sherpa (LO), ALPGEN (LO) and BlackHat+Sherpa (NLO) measured with the ATLAS detector. 


\section{Associated production of a $Z$ boson and $b$ jets in CMS}

The associated production of a $\mathrm{Z}$ boson and jets originated from the hadronization of $\mathrm{a} b$ quark is $\mathrm{a}$ unique subprocess of the $\mathrm{V}+$ jets events. The $\mathrm{Z}+\mathrm{b}$ final state is a dominant background for the Higgs production in the HZ mode and it appears in the final state of many beyond the Standard Model models processes. The $\mathrm{Z}+\mathrm{b}$ final state is also essential to understand the $\mathrm{b}$ quark production mechanism in high energy proton collisions, allowing to test the so called 4-flavour scheme (4FS), with massive b quarks in the final state, and the 5-flavour scheme (5FS), with massless b quarks in the initial state. The $\mathrm{Z}+\mathrm{b}$ differential cross sections have been measured by CMS with $20 \mathrm{fb}^{-1}$ in $8 \mathrm{TeV}$ collisions [15]. The b-jets are identified with a b-tagging technique that exploits the characteristics of the secondary vertex reconstruction, and must pass a threshold in $\mathrm{p}_{\mathrm{T}}$ of $30 \mathrm{GeV}$ and pseudorapidity less than 2.4. The $\mathrm{Z}$ boson decay products are selected using the same $\mathrm{Z}+$ jets criteria. The dominant top-induced background is evaluated in data, and the true fraction of bottom, charm and light flavours is extracted by fitting the secondary vertex mass distribution. Data ara unfolded using the SVD algorithm and the results are compared to the theoretical calculations in the 4- and 5-flavour schemes in pQCD made with MadGraph+Pythia6, and the 5-flavour scheme prediction of Powheg [16] (NLO for the leading jet). Differential cross section are presented as a function of the leading b-jet $\mathrm{pt}$ and as a function of the invariant mass of the bb system (Fig. 6). An overall normalization discrepancy of about $20 \%$ is seen in the 4-flavour scheme for the b-jet spectrum, while the NLO prediction of Powheg correctly describes the data. The 5-flavour scheme appears to overestimate data in the soft Z momentum region. Good agreement is seen in the bb invariant mass distribution comparing data with all the predictions.
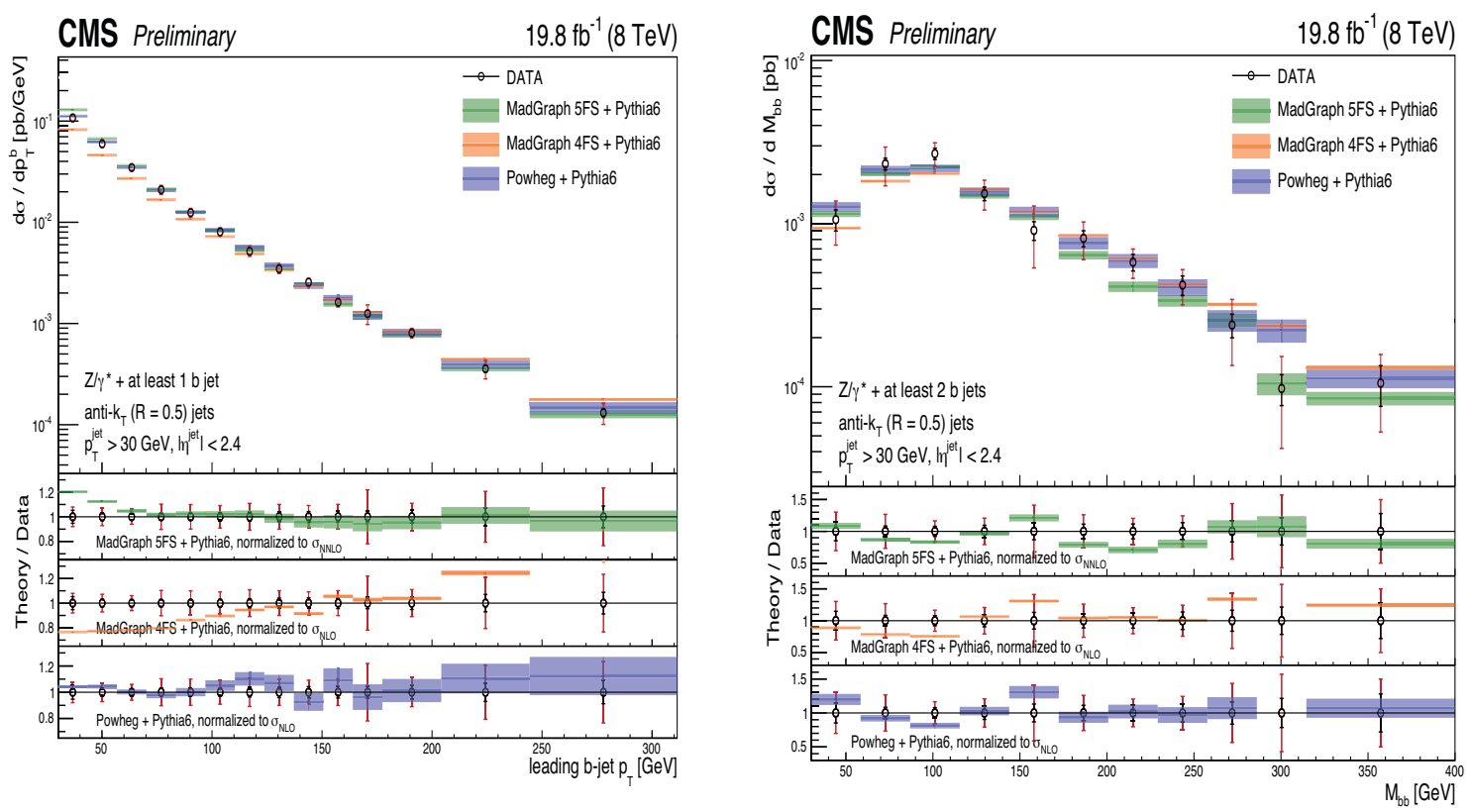

Figure 6. Differential $\mathrm{Z}+\mathrm{b}$ cross sections as a function of the leading b-jet transverse momentum (left) and as a function of the bb invariant mass compared to the theoretical predictions of MadGraph 4FS and 5FS (LO) and Powheg (NLO for the leading jet) with the CMS detector. 


\section{Conclusions}

Differential cross sections for the associated production of a $\mathrm{W}$ or $\mathrm{Z}$ boson and jets have been measured in the ATLAS and CMS experiments at a center of mass energy of 7,8 and $13 \mathrm{TeV}$ in proton-proton collisions at LHC. The $\mathrm{W}$ boson plus jets has been measured in ATLAS and CMS at 7 $\mathrm{TeV}$ and compared to several LO and NLO predictions; the Z+jets has been measured by CMS at 8 $\mathrm{TeV}$, showing also the double-differential cross sections as a function of the momentum and rapidity of the leading jet, and in ATLAS at $13 \mathrm{TeV}$. The ratio between $\mathrm{W}$ and $\mathrm{Z}$ bosons plus with at least one associated jet has been measured has been measured as a function of the leading jet momentum and compared to the NLO calculations. The $\mathrm{Z}+\mathrm{b}$ differential cross sections has been measured by CMS as a function of the most energetic $b$-jet momentum and as a function of the invariant mass of the leading and sub-leading $\mathrm{b}$ jet. The results have been compared to the LO predictions in the 4FS and 5FS and the NLO predictions for the first jet. All the differential cross sections measured in data presented have been unfolded to particle level in order to be compared to the theoretical predictions in perturbative QCD, allowing a test of the theoretical interpretation with collision data.

\section{References}

1. ATLAS Collaboration, "The ATLAS Experiment at the CERN LHC", JINST 3 (2008) S08003

2. CMS Collaboration, "The CMS experiment at the CERN LHC", JINST 3 (2008) S08004

3. ATLAS Collaboration, "Measurements of the $\mathrm{W}$ production cross sections in association with jets with the ATLAS detector", Eur.Phys.J. C75 (2015) 2, 82.

4. M. Cacciari, G. P. Salam, and G. Soyez, "The anti-k(t) jet clustering algorithm”, JHEP600 0804 (2008) 063

5. Berger, C.F. et al., "Vector Boson + Jets with BlackHat and Sherpa" Nucl.Phys.Proc.Suppl. 205-206 (2010) 92-97

6. M. L. Mangano et al., J. High Energy Phys. 0307, 001 (2003)

7. T. Gleisberg et al., J. High Energy Phys. 0902, 007 (2009)

8. CMS Collaboration, "Differential cross section measurements for the production of a W boson in association with jets in proton-proton collisions at $\sqrt{\mathrm{s}}_{\mathrm{s}}=7 \mathrm{TeV}$ ", PLB 741 (2015) 12

9. J. Alwall et al., "MadGraph 5: going beyond", JHEP 06 (2011) 128, JHEP06(2011)128

10. T. Sjostrand, S. Mrenna, and P. Skands, "PYTHIA 6.4 physics and manual", JHEP 05 (2006) 026

11. CMS Collaboration, "Measurement of the differential production cross section of $Z$ bosons in association with jets in pp collisions at $\sqrt{s}_{\mathrm{s}}=8 \mathrm{TeV}$ ", CMS-PAS-SMP-13-007

12. ATLAS Collaboration, "Measurement of the Production Cross Sections of a $Z$ boson Boson in Association with Jets in collisions at $\sqrt{s}_{\mathrm{s}}=13 \mathrm{TeV}$ with the ATLAS Detector", ATLASCONF-2015-051

13. J. Alwall et al., "The automated computation of tree-level and next-to-leading order differential cross sections, and their matching to parton shower simulations", JHEP 1407 (2014) 079

14. ATLAS Collaboration, "A measurement of the ratio of the production cross sections for $\mathrm{W}$ and $\mathrm{Z}$ bosons in association with jets with the ATLAS detector", Eur. Phys. J. C (2014) 74: 3168

15. CMS Collaboration, "Measurement of the associated production of a $Z$ boson and $b$ jets in pp collisions at $8 \mathrm{TeV}$ ", CMS-PAS-SMP-14-010

16. S. Frixione, P. Nason, and C. Oleari, "Matching NLO QCD computations with parton shower simulations: the POWHEG method", JHEP 11 (2007) 070. 